“ (C) 2017 IEEE. Personal use of this material is permitted. Permission from IEEE must be obtained for all other uses, in any current or future media, including

reprinting/republishing this material for advertising or promotional purposes, creating new collective works, for resale or redistribution to servers or lists, or reuse of any copyrighted component of this work in other works." 


\title{
An Automatic Student Verification System Utilising Off-line Thai Name Components
}

\author{
Hemmaphan Suwanwiwat \\ Information Technology Academy \\ James Cook University \\ Cairns, Australia \\ art.suwanwiwat@jcu.edu.au
}

\author{
Abhijit Das \\ School of Software \\ University of Technology Sydney \\ Sydney, Australia \\ abhijit.das@griffithuni.edu.au
}

\author{
Miguel A. Ferrer \\ IDeTIC, University of Las \\ Palmas de Gran Canaria \\ Las Palmas, Spain \\ miguelangel.ferrer@ulpgc.es
}

\author{
Umapada Pal \\ Computer Vision and Pattern \\ Recognition Unit \\ Indian Statistical Institute \\ Kolkata, India \\ umapada@isical.ac.in
}

\author{
Michael Blumenstein \\ School of Software \\ University of Technology Sydney \\ Sydney, Australia \\ Michael.Blumenstein@uts.edu.au
}

\begin{abstract}
This research proposed an automatic student identification and verification system utilising off-line Thai name components. The Thai name components consist of first and last names. Dense texture-based feature descriptors were able to yield encouraging results when applied to different handwritten text recognition scenarios. As a result, the authors employed such features in investigating their performance on Thai name component verification system. In this research, Dense-Local Binary Pattern, Dense-Local Directional Pattern, and Local Binary Pattern combined with Local Directional Pattern were employed. A base-line shape/feature i.e. Hidden Markov Model (HMM) was also utilised in this study. As there is no dataset on Thai name verification in the literature, a dataset is proposed for a Thai name verification system. The name component samples were collected from high school students. It consists of 8,400 name components (first and last names) from 100 students. Each student provided 60 genuine name components, and each of the name components was forged by 12 other students. An encouraging result was found employing the above-mentioned features on the proposed dataset.
\end{abstract}

Keywords-Student identification and verification system, Thai name components, LBP, LDP, HMM

\section{INTRODUCTION}

In general, writer identification and verification can be performed using text from a single document to identify a single author. Materials which can be used include features from handwritten words or their segmented characters from the document [1]. For the identification process, an identification system identifies the author from a list of authors known to the system using sample (which to be recognised) provided. Then for verification or authentication process, the system uses two unknown samples and verifies if the two samples belong to the same author [1].

For the proposed automatic Student Verification System, however, to be able to successfully verify students, the students first needed to be identified by their name components, once the identification process is completed, the verification process can be performed. The difference between identification and verification is the identification process concerns identify students from the given name components. Once the recognition process was completed, then the recognised exam writers were verified. This to ensure that the students who wrote the exam papers were who the claimed they are.

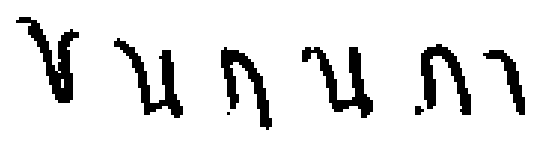

Fig. 1: An example of Thai name components

Handwriting of an individual is a pattern and every details written by and individual will hold a unique property of an individual. Name components can be found in exam papers as the students needed to write their names down to identify themselves. This also applied to examination papers in Thailand. Because younger high school students (below 16 years of age) will not be signing their names in the examination at that age. An example of Thai name components can be seen in Fig. 1. A digital image of such name components can be used for automatic offline student verification. And hence, in this study employed the name components as an approach to identify and verify each student who wrote the exam. Although, there are many forms of biometric approaches including but not limited to signature verification, fingerprint, palm print, and hand geometry [2], [3], name components can be considered an easy and optimal solution in this scenario.

Similar to signatures, some characteristics of each of the individuals' handwriting can be identified, which includes but not limited to slant, hooks, inclination, skew, and the relationship between letters are observed and later can be used to verify if the handwriting are genuine of an individual. The manual verification processes, which are carried out by Forensic Handwriting Experts or FHEs, could be a very lengthy as well 
as could be error prone for larger population sizes. As a result, an automatic verification system might be helpful.

This research proposed an automatic Student Verification System (SVS) using student name components. This research was an extension from Student Identification Systems (SIS) which were proposed in [4], [5]; the SISs were developed to identify students, so that their marks which were produced from handwritten short answer assessment system [6] can be recorded accordingly. In [4], [5], and [6], student name components were used to identify students, the student numbers were not used as it was planned that the verification system using the name components could be developed. Each student's name components consist of first and last names. The proposed system can be employed to identify who wrote the exam and then verify if the student who wrote an examination was really the person who own the name and not someone else. A successful system will benefit education sector as it can be used to verify exam attendees.

The followings are the purposes of this research:

1) To explore the characteristics of the Thai name components. Also to investigate, the viability and baseline the verification performance of Thai name components.

2) To develope of a Thai name component dataset which will be available publicly.

The remainder of this paper is organised as follows. In Section II, a brief literature was reviewed. The proposed dataset and research methodology utilised in this research can be found in Section III, while the results attained and the discussion are described in Section IV. Conclusions and future research can be found in Section V.

\section{LITERATURE REVIEW}

No work has been found on Thai name component verification up-to-date. There are, however, several Automatic Signature Verification systems (ASVs). LDP and LBP can be considered suitable for signature verification systems as a number of studies had employed such features successfully as well as robustly. Signature verification systems proposed by [7] and [8] employed text-based measures by using rotation invariant uniform LBP on the first study [7] and the latter study [8] was using LBP together with LDP features in conjunction with on binarised signature images, their experiments were conducted using MCYT75, GPDS300 and GPDS960 signature corpuses. The full results can be found in [7] and [8]. An automatic assessment mark entry system was also employed LBP and salient structural features on their numerical samples [9], the authors reported encouraging results when combined the two techniques together.

In [9], the study was conducted on a discrete HMM which was used to model each signer's features to making assumptions on the form of the underlying distribution; the signatures were modelled by two left-to-right HMM. Their experiments were performed employing HMM, SVM, and Euclidean distance classifiers. It was reported that the best results were achieved when HMM classifier was employed.

Feature extraction techniques namely, Dense-Local Binary Pattern (D-LBP), Dense-Local Directional Pattern (D-LDP), the combination of Local Binary Pattern (LBP) and Local Directional Pattern (LDP), a base-line shape/ feature-based Hidden Markov Model (HMM) were utilised to investigate the recognition and verification efficiency on hand-written student name component samples. It should be noted that Thai language has different characteristics when compared to English language in many aspects. These unique characteristic will be discussed further in Section II.

The choices of feature extraction techniques and classifiers were based on the fact that they were proven to be successful for signature verification tasks. However, they had never been employed with name components which have different characteristics in nature when compared to signature images. In this study, the exploration on the efficiency of such techniques and classifiers on handwritten name components was taken place. In other words, this study reports the efficiency on student name component verification system using such methods rather than on the signature verification system.

Since the natures of the handwritten name components and signature are considered quite different (see Section II), the authors were inspired to conduct the study. A successful SVS would benefit education sector by reducing time consumed in verifying students who sat in exams, as well as reduce human error in identifying and verifying the students. It can be concluded from the above discussion that the name entities are patterns for each individual composed of first and last names which can be used for student verification. It can also be concluded best of the authors' knowledge that there was no work conducted in this subject which was the reason this work was conceived.

\section{Proposed Thai Student NAme Component Datasets AND VERIFICATION RESEARCH METHODOLOGY}

There is no publicly available dataset of Thai language handwritten name components with forged samples; as a result, a data collection process was performed to create a custom dataset. The dataset collected for the proposed system is the first database of its type in the Thai language.

In the research proposed here, the student verification was based on one writer per name components. Upon request, the database is available for download to the research community.

\section{A. Data collection}

The Thai name components, both genuine and skilfully forged, were obtained from 100 students, whose ages were between 12 and 16 years old. Each student was asked to write their name (first and last name) 30 times, using the motion time interval technique, on white paper in the given space; they were asked to write their names as they normally do. They were asked to write ten signatures at a time, and then take a rest. After a short moment of rest, they were asked to repeat the process two more times.

In total, there were $6,000(100$ students $\times 2$ name components $\times 30$ times) genuine name components obtained. For each of the genuine name components, 12 skilfully forged name components were produced. In total, there were 2,400 (100 students $\times 2$ name components $\times 12$ times) skilfully forged name components. Altogether, there were 8,400 name 
components in this collection. Examples of genuine and skilfully forged Thai name components can be seen in Table 1. All samples were scanned at 300 dpi and stored in grey-level format, then were binarised and saved in Portable Network Graphics (PNG) format.

TABLE 1: Examples of genuine and skilfully forged Thai name components

\begin{tabular}{|c|c|c|c|}
\hline \multicolumn{2}{|c|}{ Genuine Name Components } & \multicolumn{2}{|c|}{ Forged Name Components } \\
\hline First Name & Last Name & First Name & Last Name \\
\hline หาศติสุด & 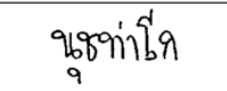 & & 1769 \\
\hline ชันร่มนี่ส & 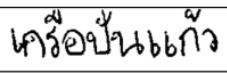 & ชนสำน์ส & 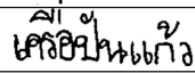 \\
\hline
\end{tabular}

\section{B. The Nature of Thai Language}

Thai language is considered very different from the other languages. It consists of 44 consonants, 18 vowels, 4 voice tones, and 3 special symbols (refer to Table 1). Altogether, there are up to 69 characters (excluding Thai numerals) in the Thai language [4]. Another aspect to mention here is zoning. Normally, the Thai language structure can be classified into four main levels which are upper zone 2, upper zone 1, middle zone, and lower zone (see Fig. 2).

The heads of the characters are important aspects of Thai language. Heads are simply small loops. They are important because many of the characters look the same without them. Only the position or whether or not the head is present will indicate what character it is. The heads of a character can be found in various locations. Thai characters can be divided into three types according to the head [4], which are:

1) No-head character, for example, "ก" for a consonant, "?" for a vowel, and "I" for a voice tone mark.

2) One-head character, for example, “ข”, “ผ”, “ล”, “อ” for consonants, and "I" for a vowel.

3) Two-head character, for example, “ณ”, “ฮ”, “พ”, and “ฒ” for consonants, and "ะ” for a vowel.

A clear example that shows the importance of the head is these three different characters that are only differentiated because of their heads: “ก", "ภ" and “ถ”. Heads of characters can be found in many positions, including upper left part of a character (“บ"), upper-right part of a character ("ห"), middle part of a character (“ฒ”), lower left part of a character (“ม”), lower right part of character ("น") [4].

TABLE 2: Thai Character set

\begin{tabular}{|c|c|}
\hline Type & Type Members \\
\hline Consonant & 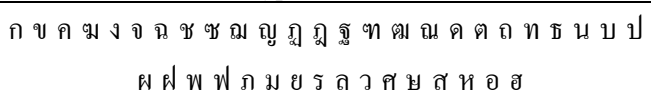 \\
\hline Vowels & $\begin{array}{c}\text { ะ า อิ อี อึ อื อุ อู เ แ โ ไ ใ อั อ็ อำ ภ (where อ can be any } \\
\text { other consonant) }\end{array}$ \\
\hline $\begin{array}{l}\text { Special } \\
\text { Symbols }\end{array}$ & อ์ ๆ ๆ (where อ can be any other consonant) \\
\hline Tones & อ่ อ้ อ๊ อ๋ (where อ can be any other consonant) \\
\hline
\end{tabular}

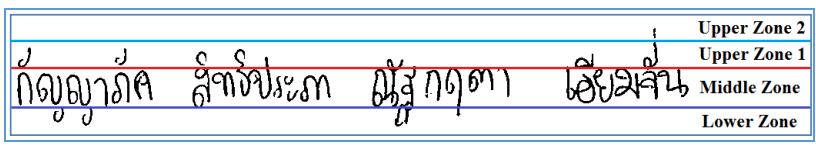

Fig. 2: Thai name component properties in zones

\section{Feature Extraction Techniques and Classifiers}

Feature extraction is one of the most crucial components in any pattern recognition approaches. The objective of feature extraction is to extract the salient information that needs to be applied in the recognition process. It reduces data by determining certain feature properties that distinguish input patterns [11].

It was found that the Thai signature identification system proposed by [12] relied heavily on global features which concerned shapes of Thai signatures, and grid features involving the overall signature appearance information. For global features, such as the signature area, net width, net height, ratio of the signature image, base-line shift, horizontal and vertical centres, maximum number of black pixels in each column, largest value of the number of black pixels of all columns, maximum number of black pixels in each row, and the largest value of the number of black pixels of all rows were used. The Grid feature was employed for signature image density. Local features such as blob, strokes, and textures can also be important features that need to be considered for Thai signatures as they are found prominently in the literature and are also visually prominent in the collected data.

It is important to note that in those studies, the signatures seemed to be name component look alike rather than signature look alike.

As described in in sub-section II.B, Thai name components are rich in local and global features. As a result, both types of features to baseline their performance were employed. There were three texture features, namely, LBP and LDP combined, Dense-LDP, and Dense-LBP employed in the experiments. A Least Square Support Vector Machine (LS-SVM) with an RBF kernel has been used as the classifier. HMM classifier was also employed in this study. The explanation of each of the employed techniques and classifiers are listed and described as follows:

1) The $L B P+L D P$ combined: Local Binary Patterns [13] operator has been used for static name component parameterisation. The grey-level image is transformed into a code matrix that is divided into 4 equal vertical blocks and 3 equal horizontal blocks, which overlaps by $60 \%$. From each block, we calculated the 255-bin histograms and the features were obtained and concatenated. A Least Square Support Vector Machine (LS-SVM) with an RBF kernel has been used as the classifier.

Local Directional Pattern [14] computes the edge response values in different directions and uses these to encode the image texture. Considering the relative edge response values in different directions, the proposed LDP feature encodes the local neighbourhood properties of the image pixels with a binary bit sequence. With this technique, the LBP and LDP were merged together in order to extract features, which were later on used in the verification process. 
2) Dense-LDP: The local descriptor method applied in this paper for featuring the traits we considered Multi-Scale Local Derivative Pattern (SPMS-LDP). From an observation, it was found that the name attributes are rich in both global and local feature. The feature SPMS-LDP is rich in both local and the global features as the total image and the image divided into different planes are considered for the scenario.

Multi-scale of four and third order of the LDP was employed here. Ten different spatial planes were considered for featuring. Each histogram distribution of bin size of 256 were calculated for each plane, ordered and spatial plane, and concatenated to get the total feature of 30,720 dimensions. This was calculated as below.

$$
\mathrm{FD}=\mathrm{NS} \times \mathrm{NO} \times 256 \times \mathrm{NSP}
$$

3) Dense-LBP: The local descriptor method applied in this paper for featuring the traits we considered Multi-Scale Local Binary Pattern (SPMS-LDP). Similar to D-LDP, an image in DLBP was divided and feature were extracted which was result in 10,240 dimensions. Where $\mathrm{FD}=$ feature dimension; $\mathrm{NS}=$ Number of Scale, $\mathrm{NO}=$ Number of Order, and NSP $=$ Number of Spatial Plane. The Spatial plane is a division of the image which divides the image into dense sampling plane. It is explained in the following fig. 3. The various level of the spatial division incorporates the local and the global feature of the traits.

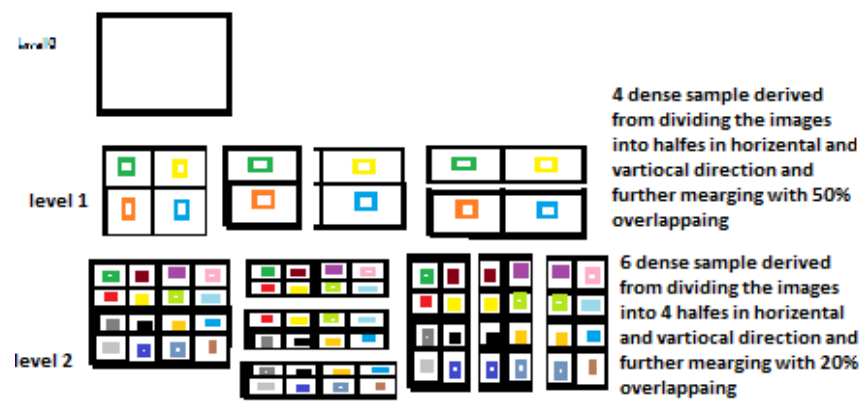

Fig. 3: The Spatial plane division to partition the image into dense sampling is explained here.

Support Vector Machines (SVMs) were employed in the experiments for a classification purpose. SVMs are one of the most popular supervised machines learning technique which performs an implicit mapping into a higher dimensional feature space. This is also known as trick of kernel. After the mapping is completed, SVMs find a linear separating hyper-plane with a maximal margin to separate data from this higher dimensional space.

The Library for Support Vector Machines (LIBSVM) was used here for the SVM implementation. SVM or LIB-SVM makes binary decision and multi-class classification for personal identification has been made in this study by adopting the oneagainst all techniques. Though there are various new kernels are available, the most frequently used kernel functions are linear, polynomial, and Radial Basis Function (RBF). This study uses the Linear kernel with SVM type-- C-SVC, kernel type - linear and cost function - 0.07. We carried out grid-search on the hyper-parameters in the 5 -fold cross validation for selecting the parameters on the training sequence. The parameter settings that produce the best cross-validation accuracy were selected.

4) A Hidden Markov Model (HMM) classifier: with geometrical features used in [10] was employed in this research. The signatures were parameterised in Cartesian and polar coordinates. Both features were combined at the score level. The Cartesian parameters consist of equidistant samples of the height and length of the signature envelope plus the number of times the vertical and horizontal line cuts the signature stroke. In polar coordinates, the parameters were equidistant samples of the envelope radius plus the stroke area in each sector. A multiobservation discrete left-to-right HMM was chosen to model each signer's features. The classification (evaluation), decoding, and training problems were solved with the forward-backward, the Viterbi, and the Baum-Welch algorithms.

\section{Datasets}

As described earlier, in total, there were 6,000 (100 students $\times 2$ name components $\times 30$ times) genuine name components in the dataset. For each of the genuine name components, 12 skilfully forged name components were produced. In total, there were 2,400 (100 students $\times 2$ name components $\times 12$ times) skilfully forged name components. As a result, there were 8,400 name components in this collection.

The training dataset contains 1,000 samples (100 students $\times$ 2 name components $\times 5$ samples), the rest of 5,000 genuine name components were used in testing dataset. All 2,400 skilfully forged name components were used for testing. Altogether, there were 7,400 samples in the testing dataset. The results of this study were given in Equal Error Rate (EER). The EER measures the error point when the false acceptance and false rejections are equal, obtaining the overlap of both the distributions. The verifiers are trained with the ten genuine name components (5 first and 5 last names) of each student in the database for repeatability of the experiments. The remainder of the 60 genuine name components and 24 skilfully forged name components of each signer were used for testing.

The EERs were obtained by getting the genuine score from the genuine samples and the forgery from the skilled forgery samples. The FAR and FRR statistics of the testing dataset are summarised in Table 3.

TABLE 3: FAR and FRR statistics of the testing dataset

\begin{tabular}{|c|c|c|}
\hline $\begin{array}{c}\text { Training samples } \\
\text { per user }\end{array}$ & $\begin{array}{c}\text { Test samples for } \\
\text { genuine }\end{array}$ & $\begin{array}{c}\text { Test samples for skilled } \\
\text { forgery }\end{array}$ \\
\hline Positive: 5 & FRR experiment: 25 & FRR experiment: 12 \\
Negative: $5 *(100-$ & FAR experiment: & FAR experiment: \\
$1)$ & $(100-1) * 25$ & $12 *(100-1)$ \\
\hline
\end{tabular}

\section{EXPERIMENTAL RESULTS}

The experiment results obtained by the aforementioned settings are shown in Table 4 . The results of verification rates of genuine and skilfully forged name components, being first and last names, are discussed as follows. It can be seen that the best verification rate of genuine first name components of $99.96 \%$ was attained when D-LBP was employed. The highest verification rate of $99.98 \%$ was also attained when the technique (D-LBP) was employed with the last name components. 
However, the verification rates for skilfully forged first and last name components were lower when compared to genuine name components. The best verification rate attained for first name components is $88.09 \%$ compared to the best recognition rate of $88.89 \%$ when the skilfully forged last name components were employed. It was found that HMM yielded the lowest verification rates when compared with the other techniques, this included both genuine and skilfully forged both for first and last name components. Using HMM, the verification rates of $80.70 \%$ was obtained, compared to the highest rate of $88.09 \%$ when D-LDP was employed with the skilfully forged first name components. In the other word, utilising HMM resulted in $7.39 \%$ lower than D-LDP.

It was also observed that on an average, the genuine first name component verification rate was $98.99 \%$ and skilfully forged first name component was $85.37 \%$. This resulted in a lower percentage of $13.62 \%$ when the skilfully forged first name components were used instead of genuine ones. Similar result was found when the genuine last name component verification rates were calculated.

For last name components, it was found that an average verification rate of $99.38 \%$ was achieved when genuine samples were employed, compared to $88.45 \%$ when the skilfully forged last name components were calculated. This yields a gap of $10.93 \%$ between the two sample types. On the average, the skilfully forged first name components yielded a lower verification rate when compared to the skilfully forged last name components by $2.69 \%$. The average verification rates of each component types can be seen in Table 5 .

It can be noted that further experiments were performed on first and last name components combined (as shown Fig. 4), however, no improvement was found. The verification rates decreased fractionally, and therefore, the results were not included in the table.

\section{ก๋ญถบภัค สุกวิปรับก \\ Fig. 4: Example of a combination of first and last name components.}

The closest work which can be used for comparison were the work proposed by [4], [5], [6], and [12]. The first three studies' results were obtained from SIS which used name components for identification process. Whereas [4] and [6] were using only Thai name components, the work in [5] was employing bilingual name components being English and Thai languages. In such work, only identification process was taken place. Feature extraction techniques used in the studies were Modified Direction Features (MDF), Gaussian Grid Feature (GGF), and Water Reservoir, Loop and Gaussian Grid Feature (WRLGGF). Because in this research, the authors were focus on verification system, the feature extraction techniques besides the ones employed [4], [5], and [6] were not utilised in this occasion, it was, however, planned to be used in the future work.

The work in [12] using global and local features. From Table 6 , it can be seen that a number of techniques were employed in the studies. For identification systems (only recognised students but not verified if the writers were students they claimed to be), it was found that the recognition rates were between $99.25 \%$ $99.52 \%$. For such systems, 100 writers were giving samples, however no forged signatures were used in the experiments.

For the studies' results reported by [12], the best accuracy rate they achieved in was $90.04 \%$ compared to the highest verification rate of $99.88 \%$ when D-LBP was employed with either genuine first or last name components, and $98.08 \%$ verification rate when D-LDP was employed with last name components. It could be noted that 600 samples which obtained from 10 writers were employed in [12] compared to 8,400 samples which obtained from 100 writers of this proposed study.

The ROC curves of the experiments are in the Fig 5. Along the $\mathrm{X}$-axis is the FAR and along the $\mathrm{Y}$-axis is the GAR. Hence it can be concluded that an appreciating verification accuracy is attended in the experiment, although there is a huge scope of development in the forgery experiments.

TABLE 4: Verification rates of genuine and skilfully forged name components with each technique used.

\begin{tabular}{|c|c|c|c|}
\hline $\begin{array}{c}\text { Techniques } \\
\text { Used }\end{array}$ & $\begin{array}{c}\text { Name Component } \\
\text { Type }\end{array}$ & $\begin{array}{c}\text { First Name } \\
(\%)\end{array}$ & $\begin{array}{c}\text { Last name } \\
(\%)\end{array}$ \\
\hline & Genuine & 96.93 & 98.01 \\
\cline { 2 - 4 } HMM & Skilled & 80.70 & 87.71 \\
\hline \multirow{3}{*}{ LBP+ LDP } & Genuine & 99.16 & 99.64 \\
\hline \multirow{3}{*}{ D-LBP } & Skilled & 84.79 & 88.88 \\
\hline \multirow{3}{*}{ D-LDP } & Genuine & 99.96 & 99.98 \\
\cline { 2 - 4 } & Skilled & 87.91 & 88.89 \\
\cline { 2 - 4 } & Genuine & 99.91 & 99.90 \\
\hline
\end{tabular}

TABLE 5: Verification rates of genuine and skilfully forged name components.

\begin{tabular}{|l|c|}
\hline Name Component Type & Average Verification Rate (\%) \\
\hline First Names - Genuine & $98.99 \%$ \\
\hline First Names - Skilled & $85.37 \%$ \\
\hline Last Names - Genuine & $99.38 \%$ \\
\hline Last Names - Skilled & $88.45 \%$ \\
\hline
\end{tabular}

TABLE 6: Comparison of verification rates attained between the proposed study and the techniques [4], [5], [6], and [12] found in the literature given the similar datasets nature.

\begin{tabular}{|l|l|}
\hline \multicolumn{1}{|c|}{ Techniques } & \multicolumn{1}{c|}{ Verification/Accuracy Rates (\%) } \\
\hline MDF/GGF [4] & $99.27 \%$ (identification - all genuine) \\
\hline WRLGGF [5] & $99.25 \%$ (bilingual - identification - all genuine) \\
\hline Enhanced MDF [6] & $99.52 \%$ (identification - all genuine) \\
\hline Features [12] & $90.04 \%$ (from 10 writers - all genuine) \\
\hline D-LBP (proposed) & $99.88 \%$ (genuine - first and last names) \\
\hline D-LDP (proposed) & $98.08 \%$ (skilfully forged -last name) \\
\hline
\end{tabular}

\section{CONCLUSIONS AND FUTURE WORK}

In this research we proposed an automatic student verification system employing off-line Thai name components. Thai name components consist of first and last names and these handwritings are rich in texture features. Dense Texture-based features descriptors were found to able to yield encouraging results when applied to different texture based environments. As a result, we employed such features in investigating their performance on Thai name components and investigate the viability of using such component for individual verification.

In this research, Dense-Local Binary Pattern (LBP), Dense- 
Local Directional Pattern (LDP), and LBP+LDP were employed. A base-line shape/feature-based Hidden Markov Model (HMM) was also utilised in this study. A novel dataset was proposed where the samples were collected from high school students. The dataset consists of 8,400 name components (first and last names) from 100 students. Each student provided 60 genuine name components, then each of the name components was forged by other 12 students. This dataset will be available for research purposes. Encouraging results were attained employing the proposed dataset. Although verification accuracy was examined in the genuine experiments, there is a large scope for further development in the forgery scenario.
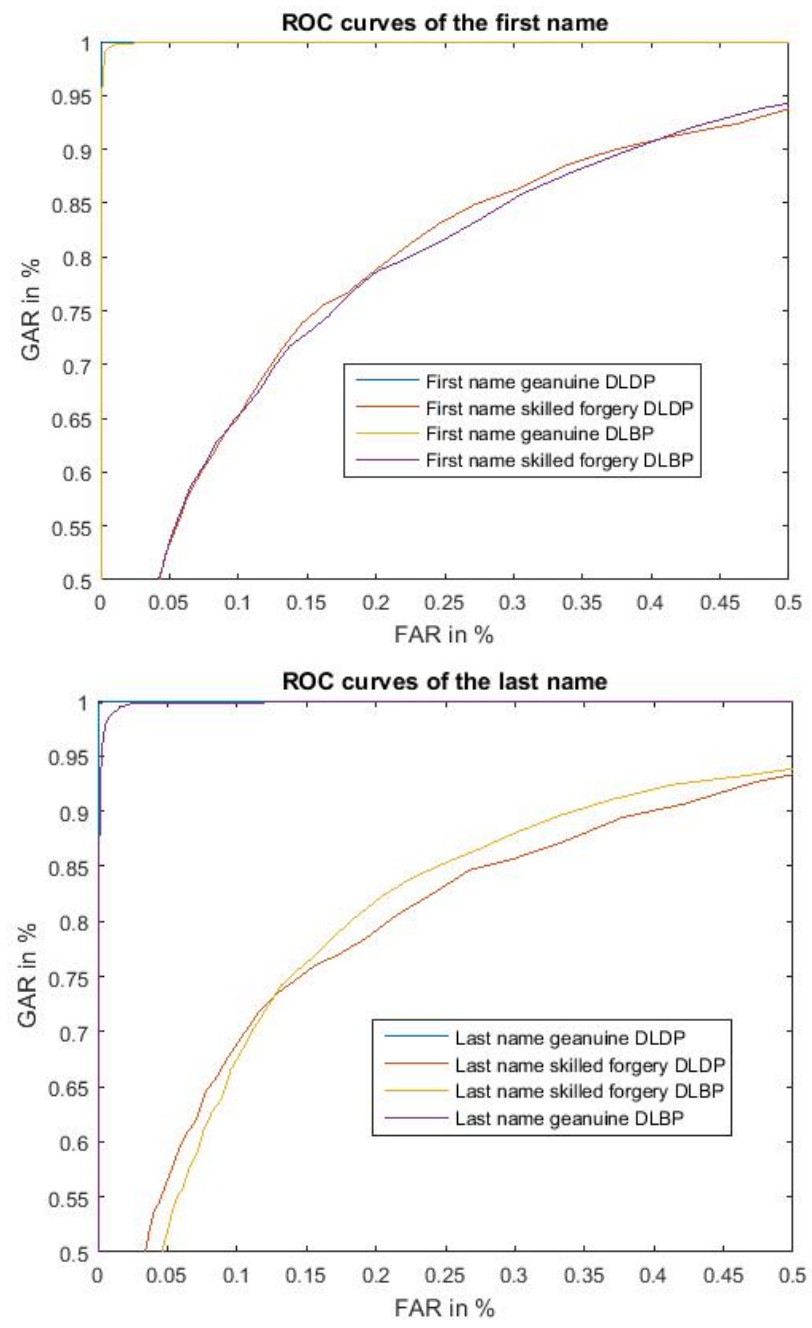

Fig. 5: ROC curves of the experiments

Future work will involve investigating name component verification in the multiscript scenario. Future work also needs to be undertaken for improvements and to enhance the performance of the proposed name component identification and verification system. More ttechniques such as deep learning may be investigated on the datasets. Larger and supplementary databases will also be created, so as a more detailed study will be performed.

\section{REFERENCES}

[1] A. Bensefia, T. Paquet, L. Heutte, A writer identification and verification system, Pattern Recognition Letters, Vol. 26, Issue 13, 2005, pp. 20802092

[2] A. Das and R. Parekh, "Iris Recognition using a Scalar based Template in Eigen-space", International Journal of Computer Science and Telecommunication, vol.3, no.5, pp 74-79., May 2012

[3] A. Das, M. A. Ferrer, U. Pal, S. Pal, M. Diaz and M. Blumenstein, "Multiscript versus single-script scenarios in automatic off-line signature verification," in IET Biometrics, vol. 5, no. 4, pp. 305-313, 122016

[4] H. Suwanwiwat, V. Nguyen, M. Blumenstein, and U. Pal, Off-line handwritten Thai name recognition for student identification in an automated assessment system, 2014 International Joint Conference on Neural Networks (IJCNN), pp.2347-2353, 2014

[5] H. Suwanwiwat, V. Nguyen, M. Blumenstein, and U. Pal, Off-Line Handwritten Bilingual Name Recognition for Student Identification in an Automated Assessment System, 14th International Conference on Frontiers in Handwriting Recognition (ICFHR 2014), pp.271-276, 2014

[6] H. Suwanwiwat, V. Nguyen, M. Blumenstein, and U. Pal, A complete automatic short answer assessment system with student identification, In 13th International Conference on Document Analysis and Recognition (ICDAR 2015), pp.611-615, 2015

[7] M. A. Ferrer, J. F. Vargas, A. Morales and A. Ordonez, "Robustness of Offline Signature Verification Based on Gray Level Features," in IEEE Transactions on Information Forensics and Security, vol. 7, no. 3, pp. 966977, June 2012

[8] M. A. Ferrer, F. Vargas, C. M. Travieso and J. B. Alonso, "Signature verification using local directional pattern (LDP)," In Proceedings of the IEEE International Carnahan Conference on Security Technology, San Jose, CA, 2010, pp. 336-340

[9] L. L. Ghai, S. B. Hisham and N. Yahya, "Automatic assessment mark entry system using local binary pattern (LBP) and salient structural features," in IEEE International Conference on Control System, Computing and Engineering (ICCSCE 2014), Batu Ferringhi, 2014, pp. 372-377

[10] M. A. Ferrer, J. B. Alonso and C. M. Travieso, "Offline geometric parameters for automatic signature verification using fixed-point arithmetic," in IEEE Transactions on Pattern Analysis and Machine Intelligence, vol. 27, no. 6, pp. 993-997, June 2005

[11] C.C. Tappert, C. Y. Suen, T. Wakahara, "The State of the Art in Online Handwriting Recognition," IEEE Transactions on Pattern Analysis and Machine Intelligence, August 1990, vol. 12, no. 8, pp. 787-808

[12] N. Chumuang and M. Ketcham. "Intelligent handwriting Thai Signature Recognition System based on artificial neuron network." TENCON 20142014 IEEE Region 10 Conference. IEEE, 2014

[13] J. Ortega-Garcia, J. Fierrez-Aguilar, D. Simon, J. Gonzalez, M. Faundez, V. Espinosa, A. Satue, I. Hernaez, J. Igarza, C. Vivaracho, D. Escudero Q. Moro, 'MCYT baseline corpus: A bimodal biometric database', in IEE Proceedings Vision, Image and Signal Processing, Special Issue on Biometrics on the Internet, vol. 150, no. 6, pp. 395-401, December 2003

[14] Md. H. Kabir, T. Jabid, O. Chae, A Local Directional Pattern Variance (LDPv) based Face Descriptor for Human Facial Expression Recognition, In Proceedings of the IEEE Advanced Video and Signal Based Surveillance (AVSS): 526-532, August 2010 